\title{
Associative Personal Information Management
}

Jonathan Diehl

RWTH Aachen University

52056 Aachen, Germany

diehl@cs.rwth-aachen.de
Copyright is held by the author/owner(s).

CHI 2009, April 4 - April 9, 2009, Boston, MA, USA

ACM 978-1-60558-247-4/08/04.

\begin{abstract}
Personal information management (PIM) is an important and hard research problem. Previous systems suffer inflexibility because of strict hierarchies and immobility. I present an alternative approach, based on associations and moving beyond today's desktop metaphor, to provide ways of managing information while mobile. To illustrate the concepts, I introduce the Associative PDA, a prototype we have designed and evaluated. Finally, I discuss some design principles, which will guide my future work.
\end{abstract}

\section{Keywords}

Personal information management, mobile, associations, associative network.

\section{ACM Classification Keywords}

H.5.m. Information Interfaces and Presentation (e.g., HCI): Miscellaneous, H.3.3. Information Systems:

Information Search and Retrieval.

\section{Introduction}

The study of personal information management (PIM) investigates the capturing, storing, and retrieving of information that is relevant for our everyday lives.

There has been much research effort in two distinct directions: understanding the human psychology 
behind PIM and designing new systems to support PIM. Nevertheless, many people still struggle today with their daily information load because PIM is a hard problem for many reasons:

- The utilized information is personal, which makes it hard to find participants for studies or to convince users to switch to alternative systems.

- When capturing information it is impossible to decide when or whether it will be used again.

- The amount of information is vast and constantly increasing [3].

- Activities of PIM are context-sensitive [1].

- Users employ multiple strategies [3].

- PIM is subjective [2].

- PIM happens everywhere [13]

To address all of these issues, I want to design a system that employs a new organization method with a mobile interface that goes beyond today's desktop metaphor.

Classic PIM systems use hierarchies to organize information, despite many researchers (e.g. [10]) having expressed their concerns. Some newer systems augment hierarchies with more flexible organization methods but there is no complete system that moves entirely beyond the use of hierarchies. I want to do so by using an associative network to organize all information and give the users more freedom in employing their own system.
Most of today's interfaces are based on the desktop metaphor. This metaphor does not fit a mobile system because of limited screen estate and direct touch interaction. I want to move beyond the desktop metaphor and create an organic interface for PIM that concentrates on the goals and activities of the user to create a natural work experience. This interface will not rely on files or applications to interact with information but instead base itself on the information and its organization.

\section{Related Work}

The Personal Brain ${ }^{1}$ and Popcorn [5] are systems for organizing knowledge in an associative network. Both visualize the network as a graph, which can be traversed to find information. The goal of these systems is to store and access thoughts, which is an important PIM activity. Nevertheless, they largely ignore other activities such as scheduling or communicating.

Semex [4] and Liaison [11] both augment existing hierarchical systems with associations to provide new ways to access the information. Semex does so by combining multiple information sources in a single application, while Liaison neatly integrates itself into the Mac operating system resulting in a very elegant interface.

Gemmel et al. [9] and Myers et al. [12] present mobile solutions for PIM. With MyLifeBits, the entire life of a person is recorded and made accessible through associations. While its goal is not to be a PIM system, it can be used as such but again without support for

\footnotetext{
${ }^{1}$ www.thebrain.com
} 
planning activities. Myers et al., on the other hand, propose a complete PIM system but do not support associations. Instead, they allow grouping of arbitrary items to reflect relationships.

None of these systems meats the goals set for my project, to escape the hierarchy metaphor on a mobile system. They, however, provide valuable insights into different aspects of PIM and will guide my design in many aspects.

\section{Previous Work}

We have created and evaluated two prototypes of a mobile associative system called the Associative PDA. Here, information is stored as nodes inside an associative network. Every information item has a type and a list of keywords or Meta data, which represent the item. To organize the information, items can be associated with any other items present in the network. These associations can then be traversed to find a desired information item. In addition, the user can initiate a search by identifying items that are related to the desired item and then finding the item in the resulting subset.

For the first prototype [6], we used Adobe Flash to create a web prototype. Its design employed a circular layout, representing the network layout of the information. It supported only text-based information and simulated, but did not run on a mobile platform. We evaluated the prototype in multiple user studies and found that the use of associations to organize information is generally well-accepted and understood.

The second prototype [8] was realized on a Windows Mobile device. The design was restricted to note-taking as a representative PIM activity. To assist the creation of associations, we added location sensing via WLAN and people sensing via Bluetooth. The evaluation was conducted over 4 weeks, to get actual usage data with real information. The results confirmed the findings of the first study series in a more natural setting.

Finally, we have introduced the concept of associative information spaces [7], which allow the seamless integration of other people's information into our own. A personal information space is the entirety of all information relevant to a single person, which usually contains references to other people. If these people also organize their information in associative networks, we can extend our network with theirs. This way, we can browse and search in their networks as if they were our own. By connecting information spaces we can support collaborative PIM activities, such as sharing, communicating, or planning.

\section{Outlook}

My goal is to create and evaluate an associative, mobile information management system. To evaluate such a personal system, though, I need to convince people to use the system with their own information in realistic situations. To do so, I need to create a polished system running on an established mobile platform that creates enough incentive to be adopted by actual users. The following principles will guide me in the development:

- The system must support all PIM activities as well as important collaboration activities.

- The system must integrate seamlessly into the mobile phone. It should be able to place and receive 
calls, send and receive messages or emails, use the built-in functionality, and trigger alarms.

- The system must integrate well into existing organization systems on the desktop. This can be achieved through synchronization or by creating an adapter for the desktop to easily access and manipulate information on the mobile device.

- The interface must be natural and easy to use. It should conform to the underlying platform's standards but not be restricted by them.

With the study of such a system, I hope to discover how associations are used for PIM, in what ways mobility influences PIM behavior, and the effect of seamless collaboration on our information sharing habits.

\section{Acknowledgements}

Special thanks go to Jan Borchers for his excellent advisory and Eileen Falke for her dedicated work on the second prototype.

\section{References}

[1] Deborah K. Barreau. Context as a factor in Personal Information Management systems. J. Am. Soc. Inf. Sci., 46(5): 327-339, June 1995.

[2] Ofer Bergman, Ruth Beyth-Marom, and Rafi Nachmias. The user-subjective approach to persona information management systems, J. Am. Soc. Inf. Sci., 54(9), 872-878, June 2003.

[3] Richard Boardman, M. Angela Sasse. "Stuff goes into the computer and doesn't come out": a cross-too study of personal information management. Proceedings of CHI '04. Vienna, AU, 2004.
[4] Yuhan Cai, Xin Luna Dong, Alon Halevy, Jing Michelle Liu, and Jayant Madhavan. Personal Information Management with SEMEX. Proceedings of SIGMOD '05, New York, NY, USA, 2005.

[5] Stephen Davies, Scotty Allen, Jon Raphaelson, Emil Meng, Jake Engleman, Roger King, and Clayton Lewis. Popcorn: the personal knowledge base. Proceedings of DIS '06, New York, NY, USA, 2006.

[6] Jonathan Diehl. The Associative PDA: An Organic User Interface for Mobile Personal Information Management. Extended Abstracts of UbiComp '06. Newport Beach, USA, 2006.

[7] Jonathan Diehl, Deniz Atak, and Jan Borchers. Associative Information Spaces. Mobile Interaction with the Real World - Workshop in conjunction with Mobile HCI 2008, 73-82. BIS-Verlag, 2008.

[8] Eileen Falke, Jonathan Diehl, and Jan Borchers. The Associative PDA 2.0. Extended Abstracts of $\mathrm{CHI}$ '08, Florence, Italy, 2008.

[9] Jim Gemmell, Gordon Bell, Roger Lueder, Steven Drucker, and Curtis Wong. MyLifeBits: fulfilling the Memex vision. Proceedings of MULTIMEDIA '02, New York, USA, 2002.

[10] M. Lansdale. The Psychology of Personal Information Management. Applied Ergonomics, 19(1):55-66, March 1988.

[11] Daniel Elias Gobera Rubalcava. Liaison: Personal Information Management by Associations. Master's thesis, University of California, San Diego, USA, 2007.

[12] Robert Myers, Edwar Zapata, and Gurminder Singh. Linking information for mobile use. Proceedings of MOBILITY '07, Singapore, 2007.

[13] Karen Church and Barry Smith. Understanding mobile information needs. Extended Abstracts of Mobile HCI '08, Amsterdam, NL, 2008. 